\title{
Un Salta-Planta Flátido en Palmas, Ormenaria rufifascia (Walker) (Insecta: Hemiptera: Auchenorrhyncha: Flatidae) ${ }^{1}$
}

\section{F. W. Howard y Susan Halbert ${ }^{2}$}

\section{Introducción}

Ormenaria rufifascia (Walker) es un insecto común en algunas especies de palmas en la Florida. Aunque esta no es comúnmente una plaga seria, a veces puede causar daños estéticos indirectamente cuando grandes poblaciones producen melaza la cual puede soportar grandes cantidades de fumagina sobre las plantas hospedantes. Los adultos igual como las ninfas son insectos conspicuos que frecuentemente llaman la atención de la gente.

\section{Distribución}

La presencia de Ormenaria rufifascia ha sido reportada a través de la península de la Florida, hacia el norte hasta el sureste de Georgia (Mead 1965), y se puede asumir que está presente a través del rango del palmito y de la serenoa (eso es, a lo largo de la costa del Golfo hasta Luisiana y a lo largo de las planicies de la costa Atlántica hacia el norte hasta las Carolinas. Este también se encuentra en Cuba (Metcalf y Bruner 1948). No fue encontrado en un reconocimiento de insectos auchenorrhynchos en palmas en la República Dominicana (Howard et al. 1981), sin embargo una especie no identificada de Ormenaria fue encontrado en palma africana de aceite, Elaeis guineensis Jacq., en Santo Domingo (Abud Antón, 1982). Ormenaria rufifascia fue interceptado en California en plantas de viveros embarcadas desde la Florida (Anónimo 2003a, Anónimo 2003b), pero no es reportado como establecido en aquel estado.

\section{Descripción}

Adultos: Los adultos de aproximadamente 11 $\mathrm{mm}$ de largo son algo grandes para ser salta-plantas. Estos son altamente conspicuos cuando están en su lugar de alimentación habitual, eso es, la superficie abaxial (inferior) de las palmas de hoja en forma de abanico. Al ser lateralmente planos y de forma deltoidea ellos parecen ser como veleros coloridos diminutos. Su color básico es azul verde pálido con varias marcas naranjas y rojizas, incluyendo dos manchas en la cara cerca a la protuberancia intermedia, bandas a lo largo de las margines dorso-laterales de la cabeza, y dos líneas

1. This document is EENY-352 (IN642), one of a series of Featured Creatures from the Entomology and Nematology Department, Florida Cooperative Extension Service, Institute of Food and Agricultural Sciences, University of Florida. Published: July 2005. This document is also available on Featured Creatures Website at http://creatures.ifas.ufl.edu. Please visit the EDIS Website at http://edis.ifas.ufl.edu.

2. F. W. Howard, University of Florida and Susan Halbert, Florida Department of Agriculture and Consumer Services, Division of Plant Industry, Gainesville, FL.

The Institute of Food and Agricultural Sciences (IFAS) is an Equal Opportunity Institution authorized to provide research, educational information and other services only to individuals and institutions that function with non-discrimination with respect to race, creed, color, religion, age, disability, sex, sexual orientation, marital status, national origin, political opinions or affiliations. U.S. Department of Agriculture, Cooperative Extension Service, University of Florida, IFAS, Florida A. \& M. University Cooperative Extension Program, and Boards of County Commissioners Cooperating. Larry Arrington, Dean 
longitudinales en el protórax y mesotórax. Las alas delanteras están bordeadas de color amarillo anaranjado. Sus ojos compuestos son de color anaranjado (Mead 1965).

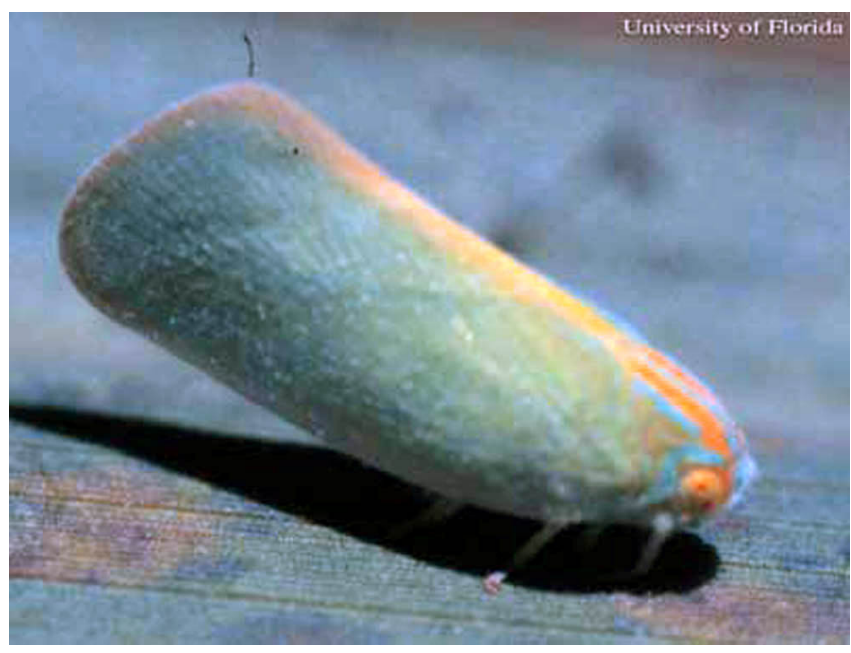

Figure 1. El adulto de Ormenaria rufifascia (Walker), un salta-planta flátido en palmas. Credits: F. W. Howard, University of Florida

Ninfas: Las ninfas son de color verde claro con líneas longitudinales amarillas borrosas. Estos colores son parcialmente enmascarados por una capa de cera flocúlenta la cual ellas secretan. Filamentos caudales de cera se arrastran detrás de ellas cuando se mueven en la superficie de la hoja.

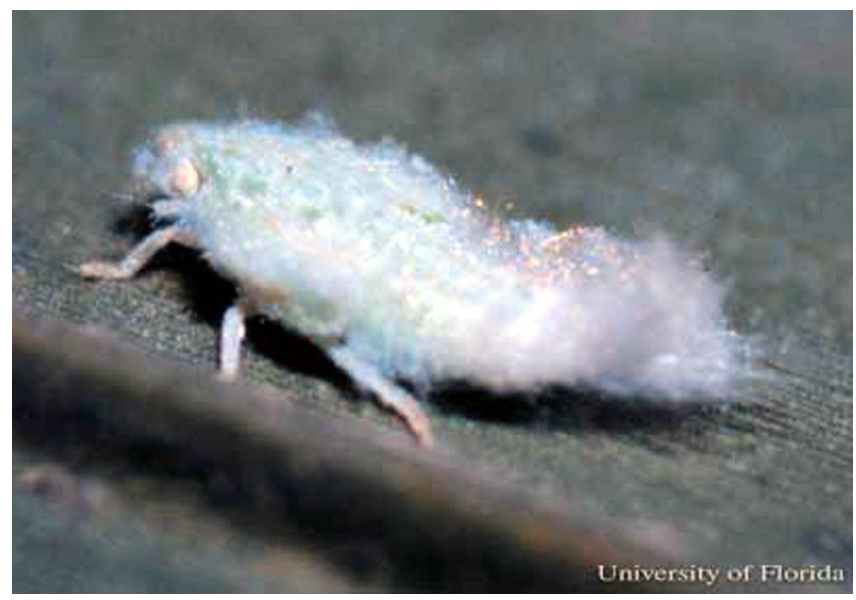

Figure 2. La ninfa de Ormenaria rufifascia (Walker), un salta-planta flátido en palmas. Credits: F. W. Howard, University of Florida

No se sabe de la existencia de otras especies de insectos con apariencia similar en las palmas de la Florida y muy pocas otras especies de Flatidae existen en palmas alrededor del mundo (Howard et al. 2001).

\section{Biología}

Ormenaria rufifascia completa una generación cada año. El estadio de huevo no ha sido observado en esta especie, pero ya que las hembras tienen un ovopositor en forma de espada el cual es típico de los insectos auchenorrhynchos, ellas probablemente depositan sus huevos dentro de los tejidos de su planta hospedante. Se cree que, como se conoce con otros insectos flátidos de Norteamérica, las hembras ovipositan en los finales del verano y los huevos nacen en enero. Las ninfas de los primeros estadios han sido vistas en las frondas de palmitos en la segunda semana de enero, y en el sur de la Florida las ninfas se han desarrollados en adultos maduros al llegar a mayo (Wilson y Tsai 1984). Estas pasan por 5 estadios durante los cuales ellas incrementan de tamaño de aproximadamente $1.4 \mathrm{~mm}$ hasta aproximadamente $4.7 \mathrm{~mm}$ de largo. El promedio del numero de días en cada estadio en palmito es 23, 20, 19, 8, y 12 (Wilson y Tsai 1984).

Los adultos igual como las ninfas son comúnmente vistos en las superficies abaxiales de las frondas de las plantas hospedantes, desde los fines de la primavera hasta los principios del verano. Durante el verano todas las ninfas maduran y solo los adultos son vistos en las palmas. Ni las ninfas ni los adultos están presentes en las palmas al finalizar el verano (Mead 1965).

Los adultos y las ninfas tienden a convivir en las hojas maduras más que en las hojas jóvenes, lo cual es característico de muchos de los insectos que se alimentan de hojas de palmas. El hecho de que las hojas mayores de las palmeras son algo horizontales y por eso ofrecen protección del sol y del viento puede ser un factor de preferencia por varias clases de insectos (Howard et al. 2001).

Las poblaciones consisten frecuentemente de pocas a varias ninfas, adultos, o ambos, aunque se han observado poblaciones densas. Un total de 50 adultos en una fronda de Pritchardia sp. fue reportada como una población inusualmente densa (Mead 1965), pero mas recientemente un total de 238 ninfas y 162 adultos fueron observados en una sola fronda de la palma Sabal maritima (Howard y Steinberg, no publicado). 


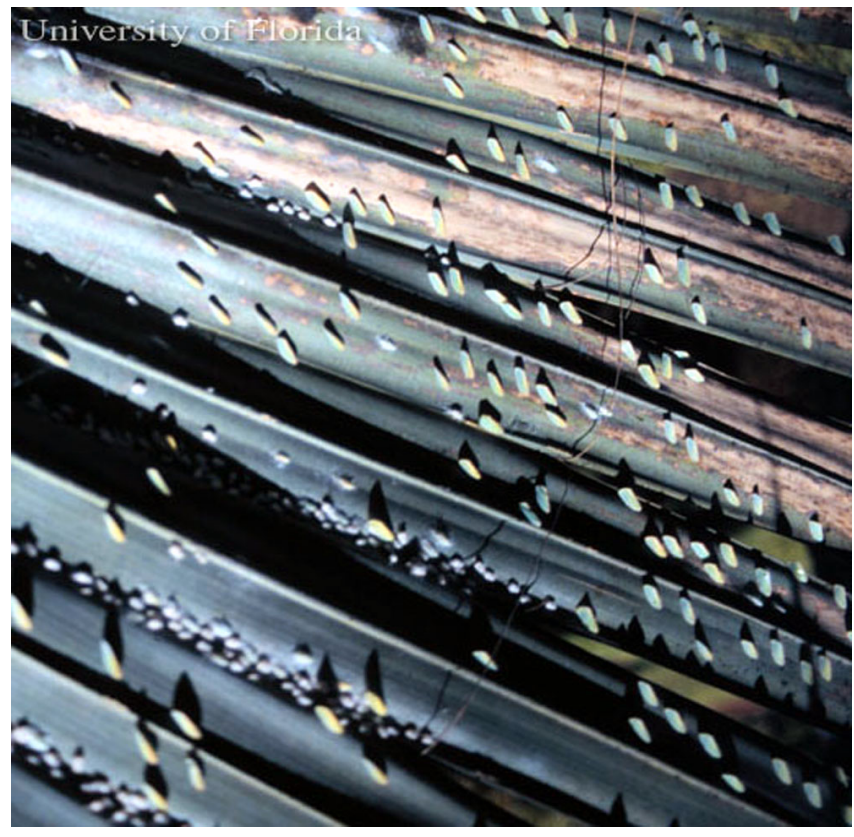

Figure 3. Una población densa de salta-hojas, Ormenaria rufifascia (Walker), sobre la fronda de una palma. Credits: F. W. Howard, University of Florida

\section{Plantas Hospederas}

Las plantas hospedantes naturales de $O$. rufifascia en la Florida aparentan ser el palmito, Sabal palmetto, y la palma enana de la Florida (serenoa, saw palmetto), Serenoa repens, ambas nativas a la Florida, pero este insecto es algunas veces visto en otras palmas. En lugares donde varias especies de palmas son cultivadas juntas, como en jardines botánicos, los adultos pueden ser observados en una gran diversidad de especies de palmas, auque posiblemente la mayoría de estas no son más que hospedantes transitorias. Las palmas, en las cuales los adultos conjuntos con las ninfas conviven, presumiblemente sustentan al insecto a través de su ciclo de vida y por esta razón pueden ser considerados como plantas hospedantes verdaderas. Las especies de palmas que entran en esta categoría están enlistadas en Cuadro 1.

Todas las especies de palmeras en la lista pertenecen a la subfamilia Coryphoideae de la familia Palmae. De las 11 especies, 9 son nativas a la Región Caribeña (cual región incluye la Florida), y dos son nativas al Hemisferio Oriental. La preponderancia de las especies americanas en esta lista talvez refleja una polarización para estas en los terrenos incluyendo los jardines botánicos en la Florida. Las frondas de

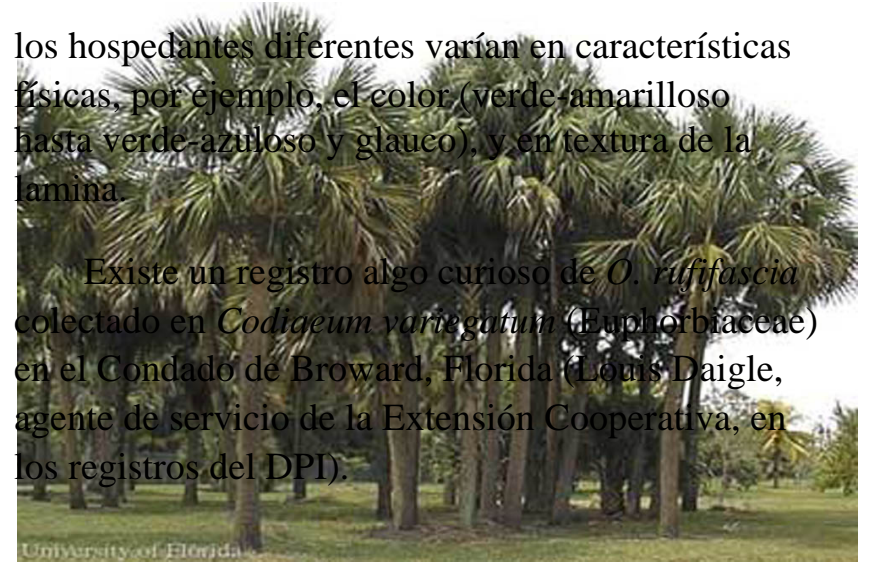

Figure 4. Palmitos, Sabal palmetto (Walt.) Loddiges. Credits: F. W. Howard, University of Florida

\section{Daño}

$\mathrm{Al}$ igual que la gran mayoría de los insectos del orden Hemiptera, O. rufifascia se alimenta succionando jugos de las plantas, y por esta razón le resta energía a la planta. Las poblaciones de este insecto usualmente consisten en pocos individuos por fronda, pero poblaciones densas como la que se le ha hecho referencia en el párrafo anterior, indudablemente consumen grandes cantidades de jugos vitales a través del tiempo. Como evidencia, las frondas que están altamente infestadas son pegajosas con melaza y sostienen capas gruesas de fumagina.

\section{Manejo}

No se ha llevado a cabo estudios para identificar los factores que regulan las poblaciones de $O$. rufifascia. Se reportaron que un pentatómido (Hemiptera: Pentatomidae) ataca O. rufifascia (Mead y Richman 2000) mas probablemente este no es un enemigo natural importante de este flátido. De cualquier modo, las poblaciones de este insecto son generalmente esparcidas y por lo tanto las medidas de control serían poco utilizadas. Esta especie no ha sido considerada como poseedora de suficiente importancia económica para justificar la investigación de métodos químicos o de otros métodos para controlarle. En casos donde los niveles de población de este insecto sean intolerables sobre unas pocas palmas ejemplares, puede ser posible controlarlos por medio de métodos físicos sencillos, como con un fuerte rocío de agua. 
Cuadro 1. Palm hosts of Ormenaria rufifascia.

\begin{tabular}{|c|c|c|c|}
\hline Species & Common Name* & Native Distribution* & Record \\
\hline $\begin{array}{l}\text { Acoelorraphe wrighti } \\
\text { (Grisbach \& H. } \\
\text { Wendland)* }\end{array}$ & $\begin{array}{l}\text { Everaglades palm, } \\
\text { Paurotis palm }\end{array}$ & $\begin{array}{l}\text { Florida, Bahamas, Cuba, Yucatan } \\
\text { Peninsula (Mexico), Caribbean } \\
\text { coast of Central America }\end{array}$ & FDACS, DPI** \\
\hline $\begin{array}{l}\text { Colpothrinax wrightii } \\
\text { (Grisebach \& H. A. } \\
\text { Wendland }\end{array}$ & Palma barrigona (Cuba) & $\begin{array}{l}\text { Pinar del Rió and Isla de la } \\
\text { Juventud, Cuba }\end{array}$ & $\begin{array}{l}\text { (Metcalf and Bruner, } \\
\text { 1948) }\end{array}$ \\
\hline $\begin{array}{l}\text { Copernicia berteroana } \\
\text { Beccari }\end{array}$ & $\begin{array}{l}\text { Yarey (Dominican } \\
\text { Republic), Dyaré (Haiti) }\end{array}$ & Hispaniola & FDACS, DPI** \\
\hline $\begin{array}{l}\text { Latania lontaroides } \\
\text { (Gaertner) }\end{array}$ & Red latan palm & Mascarene Islands & FDACS, DPI** \\
\hline $\begin{array}{l}\text { Sabal maritima (Kunth) } \\
\text { Burret }\end{array}$ & $\begin{array}{l}\text { Bull thatch (Jamaica), } \\
\text { palma cana (Cuba) }\end{array}$ & Cuba, Jamaica & $\begin{array}{l}\text { Howard and B. Steingerg } \\
\text { (unpublished) }\end{array}$ \\
\hline $\begin{array}{l}\text { Sabal palmetto (Walt.) } \\
\text { Loddiges }\end{array}$ & Cabbage palmetto & $\begin{array}{l}\text { Bahamas, western Cuba, Florida, } \\
\text { coastal regions of Georgia, and } \\
\text { North and South Carolina }\end{array}$ & FDACS, DPI** \\
\hline Sabal uresana Trelease & $\begin{array}{l}\text { Palma blanca, palma } \\
\text { cana }\end{array}$ & Chihuahua and Sonora (Mexico) & FDACS, DPI** \\
\hline $\begin{array}{l}\text { Serrenoa repens } \\
\text { (Bartram) Small }\end{array}$ & Saw palmetto & $\begin{array}{l}\text { Florida, coastal plains to Alabama } \\
\text { and Virginia }\end{array}$ & FDACS, DPI** \\
\hline
\end{tabular}

\section{Referecias}

Abud Antón AS. (1982). Plagas de insectos en Elaeis guineensis Jacq. Naturalista Postal (Carta ocasional del Herbario, Universidad de Santo Domingo. http://marcano.freeservers.com/np/np82a.html (24 June 2005).

Anonymous. 2003a. Tulare County Annual Crop and Livestock Report 2002. Tulare County Agriculture Commissioner, Tulare, CA.

Anonymous. 2003b. Santa Barbara County Agricultural Production Report 2002. Agricultural Commission, Santa Barbara County, CA, Santa Barbara, CA.
Henderson A, Galeano G, Bernal R. 1995. Field guide to the palms of the Americas Princeton University Press, Princeton, N. J.

Howard FW, Kramer JP, Féliz Peralta M. 1981. Homópteros auchenorrhyncha asociados á palmeras en un área de la República Dominicana afectada por el amarillamiento letal del cocotero. Folia Entomológica Mexicana 47:37-50.

Howard FW, Moore D, Giblin-Davis RM, Abad RG. 2001. Insects on Palms CABI Publications, Wallingford, UK.

Jones D. 1995. Palms throughout the World Smithsonian Institution Press, Washington, D. C. 
Mead FW. 1965. Ormenaria rufifascia (Walker), a planthopper pest of palms (Homoptera: Flatidae). Florida Department of Agriculture, Division of Plant Industry, Gainesville, FL.

Mead FW, Richman D. (2005). Florida predatory stinkbug, Euthyrhynchus floridanus (Linnaeus) UF/IFAS Featured Creatures. http://creatures.ifas.ufl.edu/beneficial/ e_floridanus.htm (24 June 2005).

Metcalf ZP, Bruner SC. 1948. Cuban Flatidae with new species from adjacent regions. Annals of the Entomological Society of America 41:63-118.

Wilson SW, Tsai JH. 1984. Ormenaria rufifascia (Homoptera: Fulgoroidea: Flatidae): Description of nymphal instars and notes on field biology. New York Entomological Society 92:307-315. 